\title{
Searching for traveling wave solutions of nonlinear evolution equations in mathematical physics
}

Bo Huang ${ }^{1,2^{*}}$ and Shaofen $\mathrm{Xie}^{1,2}$

\author{
"Correspondence: \\ huangbo0407@126.com \\ 'LMIB-School of Mathematics and \\ Systems Science, Beihang \\ University, Beijing, 100191, China \\ ${ }^{2}$ Guangxi Key Laboratory of Hybrid \\ Computation and IC Design \\ Analysis, Nanning, 530006, China
}

\begin{abstract}
This paper deals with the analytical solutions for two models of special interest in mathematical physics, namely the $(2+1)$-dimensional generalized Calogero-Bogoyavlenskii-Schiff equation and the $(3+1)$-dimensional generalized Boiti-Leon-Manna-Pempinelli equation. Using a modified version of the Fan sub-equation method, more new exact traveling wave solutions including triangular solutions, hyperbolic function solutions, Jacobi and Weierstrass elliptic function solutions have been obtained by taking full advantage of the extended solutions of the general elliptic equation, showing that the modified Fan sub-equation method is an effective and useful tool to search for analytical solutions of high-dimensional nonlinear partial differential equations.
\end{abstract}

Keywords: mathematical physics; traveling wave solutions; Fan sub-equation method; evolution equations

\section{Introduction}

Nonlinear partial differential equations (NLPDEs) are important mathematical models to describe physical phenomena. They are also an important field in the contemporary study of nonlinear physics, especially in soliton theory. The research on the explicit solution and integrability is helpful in clarifying the movement of the matter under nonlinear interaction and plays an important role in scientifically explaining the physical phenomena. See, for example, fluid mechanics, plasma physics, optical fibers, solid state physics, chemical kinematic, chemical physics and geochemistry. In the present paper, we will consider the following two high-dimensional nonlinear equations:

$$
\alpha u_{x t}+\beta u_{x} u_{x y}+\delta u_{y} u_{x x}+u_{x x x y}=0
$$

and

$$
u_{x x x y}-3\left(u_{x} u_{y}\right)_{x}-2 u_{y t}+3 u_{y z}=0 .
$$

Equation (1.1) [1] is the $(2+1)$-dimensional generalized Calogero-Bogoyavlenskii-Schiff (GCBS) equation, where the parameter $\alpha \neq 1, \beta, \delta$ are arbitrary nonzero constants. It contains the following famous NLPDEs:

(c) The Author(s) 2018. This article is distributed under the terms of the Creative Commons Attribution 4.0 International License (http://creativecommons.org/licenses/by/4.0/), which permits unrestricted use, distribution, and reproduction in any medium, provided you give appropriate credit to the original author(s) and the source, provide a link to the Creative Commons license, and indicate if changes were made. 
(i) Bogoyavlenskii-Schiff equation

$$
4 u_{x t}+4 u_{x} u_{x y}+2 u_{y} u_{x x}+u_{x x x y}=0
$$

(ii) Breaking soliton equation

$$
u_{x t}-4 u_{x} u_{x y}-2 u_{y} u_{x x}+u_{x x x y}=0
$$

(iii) Calogero-Bogoyavlenskii-Schiff equation

$$
4 u_{x t}+8 u_{x} u_{x y}+4 u_{y} u_{x x}+u_{x x x y}=0
$$

So it is very meaningful to further study equation (1.1). In particular, we can also get the solutions of (1.3)-(1.5).

Equation (1.2) is a generalization of the Boiti-Leon-Manna-Pempinelli (GBLMP) equation. If we take $z=t$, equation (1.2) is reduced to the following BLMP equation [2, 3]:

$$
u_{x x x y}+u_{y t}-3 u_{x x} u_{y}-3 u_{x} u_{x y}=0
$$

Searching for traveling wave solutions of NLPDEs acts a pivotal part in the study of nonlinear physical phenomena. In the past decades, much effort has been spent on the construction of exact solutions of NLPDEs, and many powerful methods have been developed such as inverse scattering transform [4], Bäcklund and Darboux transform [5-7], Hirota bilinear method [8], tanh-function method and extended tanh-function method [9-11], Fan sub-equation method [12,13], $G^{\prime} / G$ method [14, 15], F-expansion method [16] and so on. Among these methods, the Fan sub-equation method not only gives a unified formation to construct various traveling wave solutions, but also provides a guideline to classify the various types of traveling wave solutions according to five parameters.

Yomba [17] and Soliman and Abdou [18] extended the Fan sub-equation method to show that the general elliptic equation can be degenerated in some special conditions to the Riccati equation, first-kind elliptic equation, and generalized Riccati equation. Recently, Zhang and Peng [19] proposed and used a modification of the Fan sub-equation method with symbolic computation to construct a series of traveling wave solutions for the $(3+1)$-dimensional potential YTSF equation. These methods show the effectiveness of the modified Fan sub-equation method in handling the solution process of NLPDEs.

Motivated by the work described in the above three papers, in this paper, using Zhang and Peng's modification of the Fan sub-equation method, we construct the exact traveling wave solutions for the above mentioned two high-dimensional nonlinear equations by taking full advantage of the extended solutions of the following general elliptic equation:

$$
\left(\frac{d \varphi(\xi)}{d \xi}\right)^{2}=h_{0}+h_{1} \varphi(\xi)+h_{2} \varphi^{2}(\xi)+h_{3} \varphi^{3}(\xi)+h_{4} \varphi^{4}(\xi)
$$

In some special cases, when $h_{0} \neq 0, h_{1} \neq 0, h_{2} \neq 0, h_{3} \neq 0$ and $h_{4} \neq 0$, there may exist three parameters $r, p$ and $q$ such that

$$
\left(\frac{d \varphi(\xi)}{d \xi}\right)^{2}=h_{0}+h_{1} \varphi(\xi)+h_{2} \varphi^{2}(\xi)+h_{3} \varphi^{3}(\xi)+h_{4} \varphi^{4}(\xi)=\left(r+p \varphi(\xi)+q \varphi^{2}(\xi)\right)^{2}
$$


Equation (1.7) is satisfied only if the following relations hold:

$$
h_{0}=r^{2}, \quad h_{1}=2 r p, \quad h_{2}=2 r q+p^{2}, \quad h_{3}=2 p q, \quad h_{4}=q^{2} .
$$

When $h_{2}=0$ and $h_{0} \neq 0, h_{1} \neq 0, h_{3} \neq 0$ and $h_{4} \neq 0$, there may exist three parameters $r, p$ and $q$ such that

$$
\left(\frac{d \varphi(\xi)}{d \xi}\right)^{2}=h_{0}+h_{1} \varphi(\xi)+h_{3} \varphi^{3}(\xi)+h_{4} \varphi^{4}(\xi)=\left(r+p \varphi(\xi)+q \varphi^{2}(\xi)\right)^{2}
$$

Equation (1.9) requires for its existence the following relations:

$$
h_{0}=r^{2}, \quad h_{1}=2 r p, \quad h_{3}=2 p q, \quad h_{4}=q^{2}, \quad p^{2}=-2 r q, \quad r q<0 .
$$

Thus, for (1.7) and (1.9), the general elliptic equation is reduced to the generalized Riccati equation [20].

When $h_{0}=h_{1}=0$, the general elliptic equation is reduced to the auxiliary ordinary equation [21]

$$
\left(\frac{d \varphi(\xi)}{d \xi}\right)^{2}=h_{2} \varphi^{2}(\xi)+h_{3} \varphi^{3}(\xi)+h_{4} \varphi^{4}(\xi)
$$

When $h_{1}=h_{3}=0$, the general elliptic equation is reduced to the elliptic equation

$$
\left(\frac{d \varphi(\xi)}{d \xi}\right)^{2}=h_{0}+h_{2} \varphi^{2}(\xi)+h_{4} \varphi^{4}(\xi)
$$

Equation (1.12) includes the Riccati equation

$$
\left(\frac{d \varphi(\xi)}{d \xi}\right)^{2}=\left(A+\varphi^{2}(\xi)\right)^{2}
$$

when $h_{0}=A^{2}, h_{2}=2 A, h_{4}=1$, and solutions of (1.13) can be deduced from those of (1.12) in the specific case where the modulus $m$ of the Jacobi elliptic functions (solutions of (1.12)) is driven to 1 and 0.

When $h_{2}=h_{4}=0$, the general elliptic equation is reduced to the following:

$$
\left(\frac{d \varphi(\xi)}{d \xi}\right)^{2}=h_{0}+h_{1} \varphi(\xi)+h_{3} \varphi^{3}(\xi)
$$

The rest of this paper is organized as follows. In Section 2, we describe and further develop Zhang and Peng's modification of the Fan sub-equation method by making good use of the extended solutions of the general elliptic equation. In Section 3, we apply the modified Fan sub-equation method to solve the above mentioned two high-dimensional nonlinear equations, and more new exact traveling wave solutions are explicitly obtained. In Section 4, some conclusions are given. 


\section{Modification of the Fan sub-equation method}

For a given NLPDE with independent variables $x=\left(x_{0}=t, x_{1}, x_{2}, \ldots, x_{m}\right)$ and dependent variable $u$,

$$
F\left(u, u_{t}, u_{x_{i}}, u_{x_{i} x_{j}}, \ldots\right)
$$

where $F$ is in general a polynomial function of its argument, and the subscripts denote the partial derivatives. By using the traveling wave transformation, (2.1) possesses the following ansatz:

$$
u=u(\xi), \quad \xi=\sum_{i=0}^{m} k_{i} x_{i}
$$

where $k_{i}(i=0, \ldots, m)$ are undetermined constants. Substituting (2.2) into (2.1) yields an ODE

$$
Q\left(u, u^{(r)}, u^{(r+1)}, \ldots\right)=0
$$

where $u^{(r)}=\frac{d^{r} u}{d \xi^{r}}, u^{(r+1)}=\frac{d^{r+1} u}{d \xi^{r+1}}, r \geq 1$, and $r$ is the least order of derivatives in the equation. To keep the solution process as simple as possible, the function $Q$ should not be a total $\xi$-derivative of another function. Otherwise, taking integration with respect to $\xi$ further reduces the transformed equation.

Further introduce

$$
u^{(r)}(\xi)=v(\xi)=\sum_{i=1}^{n} \alpha_{i} \varphi^{i}+\alpha_{0}
$$

where $\varphi=\varphi(\xi)$ satisfies (1.6), while $\alpha_{0}, \alpha_{i}(i=1,2, \ldots, n)$ are constants to be determined later.

To determine $u(\xi)$ explicitly, one may take the following four steps:

Step 1. Determine the value of $n$ by balancing the highest-order nonlinear term(s) and the highest-order partial derivative in (2.3).

Step 2. With the aid of Maple, substitute (2.4) along with (1.6) into (2.3) to derive a polynomial in $\varphi$. Set all the coefficients of the polynomial to zero to derive a set of algebraic equations for $k_{i}(i=0, \ldots, m), \alpha_{0}$ and $\alpha_{i}(i=1, \ldots, n)$.

Step 3. Apply Wu-elimination method [22] to solve the above algebraic equations derived in Step 2, which yields the values of $k_{i}(i=0, \ldots, m), \alpha_{0}$ and $\alpha_{i}(i=1, \ldots, n)$.

Step 4. Use the results obtained in the above steps to derive a series of fundamental solutions $\varphi_{l}^{\mathrm{I}}, \varphi_{l}^{\mathrm{II}}, \varphi_{l}^{\mathrm{III}}, \varphi_{l}^{\mathrm{IV}}$ and $\varphi_{l}^{\mathrm{V}}$ to (1.6) depending on the different values chosen for $h_{0}$, $h_{1}, h_{2}, h_{3}$ and $h_{4}[17,21]$. The superscripts I, II, III, IV and V determine the group of solutions, while the subscript $l$ determines the rank of the solution. Then obtain exact solutions of (2.1) by integrating each of the obtained fundamental solutions $v(\xi)$ with respect to $\xi, r$ times:

$$
u(\xi)=\int^{\xi} \int^{\xi_{r}} \cdots \int^{\xi_{2}} v\left(\xi_{1}\right) d \xi_{1} \cdots d \xi_{r-1} d \xi_{r}+\sum_{j=1}^{r} d_{j} \xi^{r-j}
$$

where $d_{j}(j=1,2, \ldots, r)$ are arbitrary constants. 


\section{Application to evolution equations from mathematical physics}

\subsection{GCBS equation}

In this subsection we consider the $(2+1)$-dimensional GCBS equation (1.1). Using transformation (2.2) (here we denote $\xi=a x+b y-\omega t$ ), we reduce (1.1) into an ODE equation in the form

$$
-a \alpha \omega u^{\prime \prime}+(\beta+\delta) a^{2} b u^{\prime} u^{\prime \prime}+a^{3} b u^{(4)}=0
$$

Integrating (3.1) once with respect to $\xi$ and setting the integration constant to zero yields

$$
-a \alpha \omega u^{\prime}+\frac{1}{2}(\beta+\delta) a^{2} b\left(u^{\prime}\right)^{2}+a^{3} b u^{(3)}=0
$$

Further setting $r=1$ and $u^{\prime}=v$, we have

$$
-a \alpha \omega v+\frac{1}{2}(\beta+\delta) a^{2} b(v)^{2}+a^{3} b v^{\prime \prime}=0 .
$$

According to Step 1, we get $2 n=n+2$; hence $n=2$. We then suppose that (3.3) has the formal solution:

$$
v=\alpha_{2} \varphi^{2}+\alpha_{1} \varphi+\alpha_{0}
$$

Substituting (3.4) along with (1.6) into (3.3) and collecting all terms with the same order of $\varphi$ together, the left-hand side of (3.3) is converted into a polynomial in $\varphi$. Setting each coefficient of the polynomial to zero, we derive a set of algebraic equations for $a, b, \omega, \alpha_{0}$, $\alpha_{1}, \alpha_{2}$ as follows:

$$
\begin{aligned}
& \varphi^{0}: a^{3} b \alpha_{1} h_{1}+4 a^{3} b \alpha_{2} h_{0}+a^{2} b \beta \alpha_{0}^{2}+a^{2} b \delta \alpha_{0}^{2}-2 a \alpha \omega \alpha_{0}=0 \\
& \varphi^{1}: a^{3} b \alpha_{1} h_{2}+3 a^{3} b \alpha_{2} h_{1}+a^{2} b \beta \alpha_{0} \alpha_{1}+a^{2} b \delta \alpha_{0} \alpha_{1}-a \alpha \omega \alpha_{1}=0 \\
& \varphi^{2}: 3 a^{3} b \alpha_{1} h_{3}+8 a^{3} b \alpha_{2} h_{2}+2 a^{2} b \beta \alpha_{0} \alpha_{2}+a^{2} b \beta \alpha_{1}{ }^{2}+2 a^{2} b \delta \alpha_{0} \alpha_{2} \\
& \quad+a^{2} b \delta \alpha_{1}^{2}-2 a \alpha \omega \alpha_{2}=0 \\
& \varphi^{3}: 2 a^{3} b \alpha_{1} h_{4}+5 a^{3} b \alpha_{2} h_{3}+a^{2} b \beta \alpha_{1} \alpha_{2}+a^{2} b \delta \alpha_{1} \alpha_{2}=0 \\
& \varphi^{4}: 12 a^{3} b \alpha_{2} h_{4}+a^{2} b \beta \alpha_{2}^{2}+a^{2} b \delta \alpha_{2}^{2}=0
\end{aligned}
$$

Solving the set of algebraic equations by using Maple, we can obtain many kinds of solutions depending on the special values chosen for $h_{i}(i=0, \ldots, 4)$.

Case I. When $h_{0}=r^{2}, h_{1}=2 r p, h_{2}=2 r q+p^{2}, h_{3}=2 p q, h_{4}=q^{2}$, from (3.5) we have

$$
\alpha_{2}=-\frac{12 a q^{2}}{\beta+\delta}, \quad \alpha_{1}=-\frac{12 a p q}{\beta+\delta}, \quad \alpha_{0}=-\frac{12 a q r}{\beta+\delta}, \quad \omega=\frac{\left(p^{2}-4 q r\right) a^{2} b}{\alpha}
$$

and

$$
\begin{aligned}
& \alpha_{2}=-\frac{12 a q^{2}}{\beta+\delta}, \quad \alpha_{1}=-\frac{12 a p q}{\beta+\delta} \\
& \alpha_{0}=-\frac{2 a\left(p^{2}+2 q r\right)}{\beta+\delta}, \quad \omega=-\frac{\left(p^{2}-4 q r\right) a^{2} b}{\alpha} .
\end{aligned}
$$


We, therefore, have

$$
v=-\frac{12 a q^{2}}{\beta+\delta} \varphi^{2}-\frac{12 a p q}{\beta+\delta} \varphi-\frac{12 a q r}{\beta+\delta}, \quad \omega=\frac{\left(p^{2}-4 q r\right) a^{2} b}{\alpha}
$$

and

$$
v=-\frac{12 a q^{2}}{\beta+\delta} \varphi^{2}-\frac{12 a p q}{\beta+\delta} \varphi-\frac{2 a\left(p^{2}+2 q r\right)}{\beta+\delta}, \quad \omega=-\frac{\left(p^{2}-4 q r\right) a^{2} b}{\alpha} .
$$

For simplification, in the rest of this paper, we introduce that

$$
M=\frac{1}{2} \sqrt{p^{2}-4 q r}, \quad N=\frac{1}{2} \sqrt{4 q r-p^{2}} .
$$

Note that $\varphi$ is one of the twenty four $\varphi_{l}^{\mathrm{I}}(l=1,2, \ldots, 24)$. For example, if we select $l=17$, by using (2.5) we can obtain the following traveling wave solutions:

$$
u_{1}(\xi)=-\frac{6 a N}{\beta+\delta}\left[\tan \left(\frac{1}{2} N \xi\right)-\cot \left(\frac{1}{2} N \xi\right)\right]+d_{1}
$$

where $\xi=a x+b y+\frac{4 N^{2} a^{2} b}{\alpha} t, d_{1}$ is an arbitrary constant;

$$
u_{2}(\xi)=-\frac{6 a N}{\beta+\delta}\left[\tan \left(\frac{1}{2} N \xi\right)-\cot \left(\frac{1}{2} N \xi\right)\right]+\frac{8 a N^{2}}{\beta+\delta} \xi+d_{1}
$$

where $\xi=a x+b y-\frac{4 N^{2} a^{2} b}{\alpha} t, d_{1}$ is an arbitrary constant.

\section{More fundamental solutions}

Using ansatz (3.9), similarly, we can obtain a series of the fundamental solutions for (1.1) as follows.

Family 1: When $p^{2}-4 q r>0$,

$$
\begin{aligned}
u_{1,2}^{\mathrm{I}}(\xi)= & \frac{12 a M}{\beta+\delta} \tanh (M \xi)-\frac{8 a M^{2}}{\beta+\delta} \xi+d_{1} ; \\
u_{2,2}^{\mathrm{I}}(\xi)= & -\frac{8 a}{\beta+\delta} M^{2} \xi+\frac{12 a M}{\beta+\delta} \operatorname{coth}(M \xi)+d_{1} ; \\
u_{3,2}^{\mathrm{I}}(\xi)= & -\frac{a}{\beta+\delta}\left[ \pm 12 p i \arctan \left(e^{2 M \xi}\right) \mp 6 p i \arctan (\sinh (2 M \xi))+8 M^{2} \xi\right. \\
& \left.+\frac{12 M i}{\cosh (2 M \xi)}-12 M \tanh (2 M \xi)\right]+d_{1} ; \\
u_{4,2}^{\mathrm{I}}(\xi)= & -\frac{a}{\beta+\delta}\left[\mp 12 p \arctan \left(e^{2 M \xi}\right) \mp 6 p \ln (\tanh (M \xi))+8 M^{2} \xi\right. \\
& -12 M \operatorname{coth}(2 M \xi) \mp \frac{12 M}{\sinh (2 M \xi)]+d_{1} ;} \\
u_{5,2}^{\mathrm{I}}(\xi)= & \frac{6 a M}{\beta+\delta}\left[\tanh \left(\frac{1}{2} M \xi\right)+\operatorname{coth}\left(\frac{1}{2} M \xi\right)\right]-\frac{8 a M^{2}}{\beta+\delta}+d_{1} ; \\
u_{6,2}^{\mathrm{I}}(\xi)= & \frac{a}{2 B(\beta+\delta)[A \sinh (2 M \xi)+B]}\left[-16 A B M^{2} \sinh (2 M \xi) \xi\right.
\end{aligned}
$$




$$
\begin{aligned}
& +24 A M\left(A+\sqrt{A^{2}+B^{2}}\right) \sinh (2 M \xi)+48 A B M \cosh ^{2}(M \xi) \\
& \left.-16 B^{2} M^{2} \xi\right]+d_{1}, \\
u_{7,2}^{\mathrm{I}}(\xi)= & \frac{a}{(A-B)(\beta+\delta)\left[2 A \cosh ^{2}(M \xi)-A+B\right]}\left[8(A-B)^{2} M^{2} \xi\right. \\
& +12 A M(A-B) \sinh (2 M \xi)+24 A M \sqrt{B^{2}-A^{2}} \cosh ^{2}(M \xi) \\
& \left.-16 A M^{2}(A-B) \cosh ^{2}(M \xi) \xi\right]+d_{1},
\end{aligned}
$$

where $A$ and $B$ are two nonzero real constants satisfying $B^{2}-A^{2}>0$;

$$
u_{8,2}^{\mathrm{I}}(\xi)=\frac{-2 a\left[L_{1}(\xi) \tanh ^{2}\left(\frac{1}{2} M \xi\right)+L_{2}(\xi) \tanh \left(\frac{1}{2} M \xi\right)+L_{1}(\xi)\right]}{p(\beta+\delta)\left[p \tanh ^{2}\left(\frac{1}{2} M \xi\right)-4 M \tanh \left(\frac{1}{2} M \xi\right)+p\right]}+d_{1},
$$

where

$$
\begin{aligned}
& L_{1}(\xi)=4 M^{2} p^{2} \xi, L_{2}(\xi)=-16 p M^{3} \xi-48 M q r ; \\
& u_{9,2}^{\mathrm{I}}(\xi)=\frac{-8 a\left[M^{3} \tanh ^{2}\left(\frac{1}{2} M \xi\right) \xi-M^{2} p \tanh \left(\frac{1}{2} M \xi\right) \xi+3 q r \tanh \left(\frac{1}{2} M \xi\right)+M^{3} \xi\right]}{(\beta+\delta)\left[M \tanh ^{2}\left(\frac{1}{2} M \xi\right)-p \tanh \left(\frac{1}{2} M \xi\right)+M\right]}+d_{1} ; \\
& u_{10,2}^{\mathrm{I}}(\xi)=\frac{96 a q r M}{(\beta+\delta)(2 M \pm p i)[(2 M \pm p i) \tanh (M \xi) \mp 2 M i-p]}-\frac{8 M^{2} a \xi}{\beta+\delta}+d_{1} ; \\
& u_{11,2}^{\mathrm{I}}(\xi)_{+}=-\frac{48 a q r M}{p(\beta+\delta)[-p \tanh (M \xi)+2 M]}-\frac{8 M^{2} a \xi}{\beta+\delta}+d_{1} ; \\
& u_{11,2}^{\mathrm{I}}(\xi)_{-}=-\frac{24 a q r}{(\beta+\delta)[2 M \tanh (M \xi)-p]}-\frac{8 M^{2} a \xi}{\beta+\delta}+d_{1} ; \\
& u_{12,2}^{\mathrm{I}}(\xi)=u_{9,2}^{\mathrm{I}}(\xi) .
\end{aligned}
$$

Family 2: When $p^{2}-4 q r<0$,

$$
\begin{aligned}
u_{13,2}^{\mathrm{I}}(\xi)= & -\frac{12 a N}{\beta+\delta} \tan (N \xi)+\frac{8 a N^{2}}{\beta+\delta} \xi+d_{1} \\
u_{14,2}^{\mathrm{I}}(\xi)= & \frac{12 a N}{\beta+\delta} \cot (N \xi)+\frac{8 a N^{2}}{\beta+\delta} \xi+d_{1} \\
u_{15,2}^{\mathrm{I}}(\xi)= & -\frac{12 a N}{\beta+\delta}[\tan (2 N \xi) \pm \sec (2 N \xi)]+\frac{8 a N^{2}}{\beta+\delta} \xi+d_{1} \\
u_{16,2}^{\mathrm{I}}(\xi)= & \frac{12 a N}{\beta+\delta}[\cot (2 N \xi) \pm \csc (2 N \xi)]+\frac{8 a N^{2}}{\beta+\delta} \xi+d_{1} \\
u_{17,2}^{\mathrm{I}}(\xi)= & \frac{6 a N}{\beta+\delta}\left[\cot \left(\frac{1}{2} N \xi\right)-\tan \left(\frac{1}{2} N \xi\right)\right]+\frac{8 a N^{2}}{\beta+\delta} \xi+d_{1} ; \\
u_{18,2}^{\mathrm{I}}(\xi)= & \frac{a}{B(\beta+\delta)[A \sin (2 N \xi)+B]}\left[8 A B N^{2} \sin (2 N \xi) \xi+24 A B N \cos ^{2}(N \xi)\right. \\
& \left.+12 A N\left(A \pm \sqrt{A^{2}-B^{2}}\right) \sin (2 N \xi)+8 B^{2} N^{2} \xi\right]+d_{1}, \\
u_{19,2}^{\mathrm{I}}(\xi)= & \frac{a}{(A-B)(\beta+\delta)[A \cos (2 N \xi)+B]}\left[ \pm 24 A N \sqrt{A^{2}-B^{2}} \cos ^{2}(N \xi)\right. \\
& \left.-12 A N(A-B) \sin (2 N \xi)+16 A N^{2}(A-B) \cos ^{2}(N \xi) \xi-8 N^{2}(A-B)^{2} \xi\right]+d_{1},
\end{aligned}
$$


where $A$ and $B$ are two nonzero real constants satisfying $A^{2}-B^{2}>0$;

$$
\begin{aligned}
& u_{20,2}^{\mathrm{I}}(\xi)=\frac{24 a q r}{(\beta+\delta)[2 N \tan (N \xi)+p]}+\frac{8 a N^{2}}{\beta+\delta} \xi+d_{1} ; \\
& u_{21,2}^{\mathrm{I}}(\xi)=\frac{48 a q r N}{p(\beta+\delta)[p \tan (N \xi)-2 N]}+\frac{8 a N^{2}}{\beta+\delta} \xi+d_{1} ; \\
& u_{22,2}^{\mathrm{I}}(\xi)=\frac{96 a q r N}{(\beta+\delta)(2 N \mp p)[(2 N \mp p) \tan (N \xi) \pm 2 N+p]}+\frac{8 a N^{2}}{\beta+\delta} \xi+d_{1} ; \\
& u_{23,2}^{\mathrm{I}}(\xi)_{+}=\frac{48 a q r N}{p(\beta+\delta)[p \tan (N \xi)-2 N]}+\frac{8 a N^{2}}{\beta+\delta} \xi+d_{1} ; \\
& u_{23,2}^{\mathrm{I}}(\xi)_{-}=\frac{24 a q r}{(\beta+\delta)[2 N \tan (N \xi)+p]}+\frac{8 a N^{2}}{\beta+\delta} \xi+d_{1} ; \\
& u_{24,2}^{\mathrm{I}}(\xi)=u_{21,2}^{\mathrm{I}}(\xi) ;
\end{aligned}
$$

where $\xi=a x+b y-\frac{4 N^{2} a^{2} b}{\alpha} t, d_{1}$ is an arbitrary constant.

Case II. When $h_{0}=r^{2}, h_{1}=2 r p, h_{2}=0, h_{3}=2 p q, h_{4}=q^{2}$ and $p^{2}=-2 r q$, from (3.5) we have

$$
\alpha_{2}=-\frac{12 a q^{2}}{\beta+\delta}, \quad \alpha_{1}=-\frac{12 a p q}{\beta+\delta}, \quad \alpha_{0}=0, \quad \omega=-\frac{3 a^{2} b p^{2}}{\alpha}
$$

and

$$
\alpha_{2}=-\frac{12 a q^{2}}{\beta+\delta}, \quad \alpha_{1}=-\frac{12 a p q}{\beta+\delta}, \quad \alpha_{0}=\frac{6 a p^{2}}{\beta+\delta}, \quad \omega=\frac{3 a^{2} b p^{2}}{\alpha}
$$

We, therefore, have

$$
v=-\frac{12 a q^{2}}{\beta+\delta} \varphi^{2}-\frac{12 a p q}{\beta+\delta} \varphi, \quad \omega=-\frac{3 a^{2} b p^{2}}{\alpha}
$$

and

$$
v=-\frac{12 a q^{2}}{\beta+\delta} \varphi^{2}-\frac{12 a p q}{\beta+\delta} \varphi+\frac{6 a p^{2}}{\beta+\delta}, \quad \omega=\frac{3 a^{2} b p^{2}}{\alpha} .
$$

Note that $\varphi$ is one of the twelve $\varphi_{l}^{\mathrm{II}}(l=1,2, \ldots, 12)$. For example, if we select $l=2$, by using (2.5), we can obtain the following traveling wave solutions:

$$
\begin{aligned}
u_{3}(\xi)= & -\frac{8 a \sqrt{-6 q r}}{\beta+\delta} \ln \left|\operatorname{coth}\left(\frac{1}{2} \sqrt{-6 q r} \xi\right)+1\right|+\frac{6 a \sqrt{-6 q r}}{\beta+\delta} \operatorname{coth}\left(\frac{1}{2} \sqrt{-6 q r} \xi\right) \\
& -\frac{4 a(2 \sqrt{-6 q r} \pm 3 \sqrt{-2 q r}-3 p)}{\beta+\delta} \ln \left|\sinh \left(\frac{1}{2} \sqrt{-6 q r \xi}\right)\right| \\
& \pm \frac{6 a p \sqrt{-2 q r}}{\beta+\delta} \xi+d_{1},
\end{aligned}
$$


where $\xi=a x+b y+\frac{3 a^{2} b p^{2}}{\alpha} t, d_{1}$ is an arbitrary constant;

$$
\begin{aligned}
u_{4}(\xi)= & -\frac{8 a \sqrt{-6 q r}}{\beta+\delta} \ln \left|\operatorname{coth}\left(\frac{1}{2} \sqrt{-6 q r} \xi\right)+1\right|+\frac{6 a \sqrt{-6 q r}}{\beta+\delta} \operatorname{coth}\left(\frac{1}{2} \sqrt{-6 q r} \xi\right) \\
& -\frac{4 a(2 \sqrt{-6 q r} \pm 3 \sqrt{-2 q r}-3 p)}{\beta+\delta} \ln \left|\sinh \left(\frac{1}{2} \sqrt{-6 q r} \xi\right)\right| \\
& +\frac{6 a p( \pm \sqrt{-2 q r}+p)}{\beta+\delta} \xi+d_{1},
\end{aligned}
$$

where $\xi=a x+b y-\frac{3 a^{2} b p^{2}}{\alpha} t, d_{1}$ is an arbitrary constant.

\section{More fundamental solutions}

Using ansatz (3.15), if we select $l=5,8$, similarly, we can obtain more fundamental solutions for (1.1) as follows.

$$
\begin{aligned}
u_{5,2}^{\mathrm{II}}(\xi)= & -\frac{12 a p}{\beta+\delta} \ln \left|\operatorname{coth}\left(\frac{1}{4} \sqrt{-6 q r} \xi\right)-\tanh \left(\frac{1}{4} \sqrt{-6 q r} \xi\right)\right| \\
& +\frac{3 a \sqrt{-6 q r}}{\beta+\delta}\left[\tanh \left(\frac{1}{4} \sqrt{-6 q r} \xi\right)+\operatorname{coth}\left(\frac{1}{4} \sqrt{-6 q r} \xi\right)\right] \\
& \mp \frac{12 a \sqrt{-2 q r}}{\beta+\delta} \ln \left[\sinh \left(\frac{1}{2} \sqrt{-6 q r} \xi\right)\right]+\frac{6 a\left(4 q r \pm p \sqrt{-2 q r}+p^{2}\right)}{\beta+\delta} \xi+d_{1} ; \\
u_{8,2}^{\mathrm{II}}(\xi)= & \frac{-2 a M(\xi)}{\sqrt{-q r}(\beta+\delta)\left(\tanh ^{2} Y+2 \sqrt{3} \tanh Y+1\right)}+d_{1},
\end{aligned}
$$

where

$$
\begin{aligned}
M(\xi)= & -6 \sqrt{-q r}(p+\sqrt{-2 q r})\left(1+\tanh ^{2} Y\right) \ln \left[\sqrt{-q r}\left(\tanh ^{2} Y+2 \sqrt{3} \tanh Y+1\right)\right] \\
& -12 \sqrt{-3 q r}(p+\sqrt{-2 q r}) \tanh Y \ln \left[\sqrt{-q r}\left(\tanh ^{2} Y+2 \sqrt{3} \tanh Y+1\right)\right] \\
& -24 \sqrt{-3 q r}(p+\sqrt{-2 q r}) \tanh Y \ln (\cosh Y) \\
& -3 \ln 2 \sqrt{-q r}(p+\sqrt{-2 q r})\left(\tanh ^{2} Y+1\right) \\
& -2 \sqrt{6}\left(p^{2}-p \sqrt{-2 q r}+4 q r\right)\left(\tanh ^{2} Y+1\right) \cdot Y \\
& -12 \sqrt{2}\left(p^{2}-p \sqrt{-2 q r}+4 q r\right) \tanh Y \cdot Y \\
& -6 \sqrt{3} \ln 2 \sqrt{-q r}(p+\sqrt{-2 q r}) \tanh Y-12 \sqrt{6} q r \tanh Y \\
& -12 \sqrt{-q r}(p+\sqrt{-2 q r})\left(\tanh ^{2} Y+1\right) \ln (\cosh Y),
\end{aligned}
$$

with $Y=\frac{1}{4} \sqrt{-6 q r} \xi, \xi=a x+b y-\frac{3 a^{2} b p^{2}}{\alpha} t, d_{1}$ is an arbitrary constant.

Case III. If $h_{0}=h_{1}=0, h_{2}, h_{3}, h_{4}$ are arbitrary constants, the system does not admit any solution of this group.

Case IV. If $h_{1}=h_{3}=0$, when $h_{2}^{2}-3 h_{0} h_{4}>0$, from (3.5) we have

$$
\begin{aligned}
& \alpha_{2}=-\frac{12 a h_{4}}{\beta+\delta}, \quad \alpha_{1}=0, \\
& \alpha_{0}=\frac{4 a\left(-h_{2} \pm \sqrt{h_{2}^{2}-3 h_{0} h_{4}}\right)}{\beta+\delta}, \quad \omega= \pm \frac{4 a^{2} b \sqrt{h_{2}^{2}-3 h_{0} h_{4}}}{\alpha} .
\end{aligned}
$$


We, therefore, have

$$
v=-\frac{12 a h_{4}}{\beta+\delta} \varphi^{2}+\frac{4 a\left(-h_{2} \pm \sqrt{h_{2}^{2}-3 h_{0} h_{4}}\right)}{\beta+\delta}, \quad \omega= \pm \frac{4 a^{2} b \sqrt{h_{2}^{2}-3 h_{0} h_{4}}}{\alpha} .
$$

Note that $\varphi$ is one of the sixteen $\varphi_{l}^{\mathrm{IV}}(l=0, \ldots, 16)$. For example, if we select $l=4$, then $h_{0}=m^{2}-1, h_{2}=2-m^{2}, h_{4}=-1$. Using (2.5), we can obtain the following traveling wave solutions:

$$
u_{5}(\xi)=\frac{12 a}{\beta+\delta} E(\phi, m)+\frac{4 a\left(m^{2}-2 \pm \sqrt{m^{4}-m^{2}+1}\right)}{\beta+\delta} \xi+d_{1}
$$

where $\xi=a x+b y \mp \frac{4 a^{2} b \sqrt{m^{4}-m^{2}+1}}{\alpha} t, E(\phi, m)$ is the second kind elliptic integral, $\phi=$ $\arcsin (s n \xi)$ is the argument of $\xi, d_{1}$ is an arbitrary constant.

In the limit case when $m \rightarrow 1$, the solutions (3.20) become

$$
u_{51}(\xi)=\frac{12 a}{\beta+\delta} \tanh (\xi)+\frac{4 a(-1 \pm 1)}{\beta+\delta} \xi+d_{1}
$$

where $\xi=a x+b y \mp \frac{4 a^{2} b}{\alpha} t, d_{1}$ is an arbitrary constant.

When $m \rightarrow 0$, the solutions (3.20) become a rational solution

$$
u_{52}(\xi)=\frac{4 a(1 \pm 1)}{\beta+\delta} \xi+d_{1}
$$

where $\xi=a x+b y \mp \frac{4 a^{2} b}{\alpha} t, d_{1}$ is an arbitrary constant.

When $h_{2}^{2}-3 h_{0} h_{4}<0$, from (3.5) we have

$$
\begin{aligned}
& \alpha_{2}=-\frac{12 a h_{4}}{\beta+\delta}, \quad \alpha_{1}=0, \\
& \alpha_{0}=\frac{-4 a\left(h_{2} \mp i \sqrt{3 h_{0} h_{4}-h_{2}^{2}}\right)}{\beta+\delta}, \quad \omega= \pm \frac{4 a^{2} b \sqrt{3 h_{0} h_{4}-h_{2}^{2}}}{\alpha} i .
\end{aligned}
$$

Note that in this case $\varphi$ may be one of $\varphi_{l}^{\mathrm{IV}}(l=1,2,13,15)$. For example, if we select $l=1$, i.e., $h_{4}=m^{2}, h_{2}=-\left(1-m^{2}\right), h_{0}=1$, which satisfy $h_{2}^{2}-3 h_{0} h_{4}<0$ for $\frac{\sqrt{7}-\sqrt{3}}{2}<m \leq 1$. Using (2.5), we can obtain the following traveling wave solutions:

$$
u_{1}^{\mathrm{IV}}(\xi)=\frac{12 a}{\beta+\delta} E(\phi, m)-\frac{4 a\left(2+m^{2} \mp i \sqrt{5 m^{2}-m^{4}-1}\right)}{\beta+\delta} \xi+d_{1}
$$

where $\xi=a x+b y \mp i \frac{4 a^{2} b \sqrt{5 m^{2}-m^{4}-1}}{\alpha} t, E(\phi, m)$ is the second kind elliptic integral, $\phi=$ $\arcsin (s n \xi)$ is the argument of $\xi, d_{1}$ is an arbitrary constant.

In the limit case when $m \rightarrow 1$, the solutions (3.23) become

$$
u_{1,1}^{\mathrm{IV}}(\xi)=\frac{12 a}{\beta+\delta} \tanh \xi-\frac{4 a(3 \mp i \sqrt{3})}{\beta+\delta} \xi+d_{1}
$$

where $\xi=a x+b y \mp i \frac{4 a^{2} b \sqrt{3}}{\alpha} t, d_{1}$ is an arbitrary constant. 
Case V. If $h_{2}=h_{4}=0, h_{3}>0, h_{0}, h_{1}$ are arbitrary constants, from (3.5) we have

$$
\alpha_{2}=0, \quad \alpha_{1}=-\frac{3 a h_{3}}{\beta+\delta}, \quad \alpha_{0}= \pm \frac{\alpha \sqrt{3 h_{1} h_{3}}}{\beta+\delta} i, \quad \omega= \pm \frac{a^{2} b \sqrt{3 h_{1} h_{3}}}{\alpha} i
$$

We, therefore, have

$$
v=-\frac{3 a h_{3}}{\beta+\delta} \varphi \pm \frac{\alpha \sqrt{3 h_{1} h_{3}}}{\beta+\delta} i, \quad \omega= \pm \frac{a^{2} b \sqrt{3 h_{1} h_{3}}}{\alpha} i
$$

Substituting the corresponding solutions [17] of (1.6) into (3.26) and using (2.5), we can obtain a Weierstrass elliptic function solution

$$
u_{6}(\xi)=-\frac{3 a h_{3}}{\beta+\delta} \int^{\xi} \wp\left(\frac{\sqrt{h_{3}}}{2} \xi_{1}, g_{2}, g_{3}\right) d \xi_{1} \pm \frac{\alpha \sqrt{3 h_{1} h_{3}} i}{\beta+\delta} \xi+d_{1}
$$

where $\xi=a x+b y \mp \frac{a^{2} b \sqrt{3 h_{1} h_{3}} i}{\alpha} t, g_{2}=-\frac{4 h_{1}}{h_{3}}, g_{3}=-\frac{4 h_{0}}{h_{3}}, d_{1}$ is an arbitrary constant.

Remark 3.1 In this case (in the condition of (1.14)), the solution is a Weierstrass elliptic doubly periodic type solution only for $h_{3}>0$, so we just consider the case $h_{3}>0$. If $h_{1}<0$, then imaginary number $i$ appears again in (3.25), (3.26) and (3.27), and then other steps can be considered in a similar way.

\subsection{GBLMP equation}

In this subsection we consider the $(3+1)$-dimensional GBLMP equation (1.2). Note that since each term of equation (1.2) contains a partial derivative with respect to $y$, for the convenience of calculation, we denote transformation (2.2) by $\xi=a x+y+c z-\omega t$, then we reduce (1.2) into an ODE equation in the form

$$
a^{3} u^{(4)}-6 a^{2} u^{\prime} u^{\prime \prime}+2 \omega u^{\prime \prime}+3 c u^{\prime \prime}=0
$$

Integrating (3.28) once with respect to $\xi$ and setting the integration constant to zero yields

$$
a^{3} u^{(3)}-3 a^{2}\left(u^{\prime}\right)^{2}+(2 \omega+3 c) u^{\prime}=0
$$

Further setting $r=1$ and $u^{\prime}=v$, we have

$$
a^{3} v^{\prime \prime}-3 a^{2}(v)^{2}+(2 \omega+3 c) v=0
$$

According to Step 1 , we get $2 n=n+2$; hence $n=2$. We then suppose that (3.30) has the formal solution

$$
v=\alpha_{2} \varphi^{2}+\alpha_{1} \varphi+\alpha_{0}
$$

Substituting (3.31) along with (1.6) into (3.30) and collecting all terms with the same order of $\varphi$ together, the left-hand side of (3.30) is converted into a polynomial in $\varphi$. Setting 
each coefficient of the polynomial to zero, we derive a set of algebraic equations for $a, c$, $\omega, \alpha_{0}, \alpha_{1}, \alpha_{2}$ as follows:

$$
\begin{aligned}
& \varphi^{0}: a^{3} \alpha_{1} h_{1}+4 a^{3} \alpha_{2} h_{0}-6 a^{2} \alpha_{0}^{2}+6 c \alpha_{0}+4 \omega \alpha_{0}=0, \\
& \varphi^{1}: a^{3} \alpha_{1} h_{2}+3 a^{3} \alpha_{2} h_{1}-6 a^{2} \alpha_{0} \alpha_{1}+3 c \alpha_{1}+2 \omega \alpha_{1}=0, \\
& \varphi^{2}: 3 a^{3} \alpha_{1} h_{3}+8 a^{3} \alpha_{2} h_{2}-12 a^{2} \alpha_{0} \alpha_{2}-6 a^{2} \alpha_{1}^{2}+6 c \alpha_{2}+4 \omega \alpha_{2}=0, \\
& \varphi^{3}: 2 a^{3} \alpha_{1} h_{4}+5 a^{3} \alpha_{2} h_{3}-6 a^{2} \alpha_{1} \alpha_{2}=0, \\
& \varphi^{4}: 2 a^{3} \alpha_{2} h_{4}-a^{2} \alpha_{2}^{2}=0 .
\end{aligned}
$$

Solving the set of algebraic equations by using Maple, similarly, we can get the solutions $\alpha_{2}, \alpha_{1}, \alpha_{0}$ and $\omega$ depending on the special values chosen for $h_{i}(i=0, \ldots, 4)$. Note that $\varphi(\xi)$ intervening in (3.31) may be one of $\varphi_{l}^{\mathrm{I}}(\xi)$ or $\varphi_{l}^{\mathrm{II}}(\xi)$ or $\varphi_{l}^{\mathrm{IV}}(\xi)$ or $\varphi_{l}^{\mathrm{V}}(\xi)$, using (2.5) we can have many kinds of solutions. For the limit of length, we only consider Case I here.

When $h_{0}=r^{2}, h_{1}=2 r p, h_{2}=2 r q+p^{2}, h_{3}=2 p q, h_{4}=q^{2}$, from (3.32) we have

$$
\begin{aligned}
& \alpha_{2}=2 a q^{2}, \quad \alpha_{1}=2 a p q, \\
& \alpha_{0}=2 a q r, \quad \omega=-\frac{1}{2} a^{3} p^{2}+2 a^{3} q r-\frac{3}{2} c
\end{aligned}
$$

and

$$
\begin{aligned}
& \alpha_{2}=2 a q^{2}, \quad \alpha_{1}=2 a p q, \\
& \alpha_{0}=\frac{1}{3} a p^{2}+\frac{2}{3} a q r, \quad \omega=\frac{1}{2} a^{3} p^{2}-2 a^{3} q r-\frac{3}{2} c .
\end{aligned}
$$

We, therefore, have

$$
v=2 a q^{2} \varphi^{2}+2 a p q \varphi+2 a q r, \quad \omega=-\frac{1}{2} a^{3} p^{2}+2 a^{3} q r-\frac{3}{2} c
$$

and

$$
v=2 a q^{2} \varphi^{2}+2 a p q \varphi+\frac{1}{3} a p^{2}+\frac{2}{3} a q r, \quad \omega=\frac{1}{2} a^{3} p^{2}-2 a^{3} q r-\frac{3}{2} c .
$$

Note that $\varphi$ is one of the twenty four $\varphi_{l}^{\mathrm{I}}(l=1,2, \ldots, 24)$. For example, if we select $l=5$, by using (2.5) we can obtain the following traveling wave solutions:

$$
u_{7}(\xi)=-a M\left[\tanh \left(\frac{1}{2} M \xi\right)+\operatorname{coth}\left(\frac{1}{2} M \xi\right)\right]+d_{1},
$$

where $\xi=a x+y+c z+\left(\frac{1}{2} a^{3} p^{2}-2 a^{3} q r+\frac{3}{2} c\right) t, d_{1}$ is an arbitrary constant;

$$
u_{8}(\xi)=-a M\left[\tanh \left(\frac{1}{2} M \xi\right)+\operatorname{coth}\left(\frac{1}{2} M \xi\right)\right]+\frac{4 a M^{2}}{3} \xi+d_{1},
$$

where $\xi=a x+y+c z-\left(\frac{1}{2} a^{3} p^{2}-2 a^{3} q r-\frac{3}{2} c\right) t, d_{1}$ is an arbitrary constant. 


\section{More fundamental solutions}

Using ansatz (3.36), if we select $l=2,8,20$, similarly, we can obtain more fundamental solutions for (1.2) as follows.

$$
\begin{aligned}
& u u_{2,2}^{\mathrm{I}}(\xi)=-2 a M \operatorname{coth}(M \xi)+\frac{1}{3} a p^{2} \xi-\frac{4}{3} a q r \xi+d_{1} \\
& u u_{8,2}^{\mathrm{I}}(\xi)=\frac{-a N(\xi)}{6 p M\left(p \tanh ^{2} Y-4 M \tanh Y+p\right)}+d_{1}
\end{aligned}
$$

where

$$
\begin{aligned}
N(\xi)= & -4 p^{2}\left(p^{2}+8 q r\right) \tanh ^{2} Y \cdot Y+16 p M\left(p^{2}+8 q r\right) \tanh Y \cdot Y \\
& +96 q r M^{2} \tanh Y-4 p^{2}\left(p^{2}+8 q r\right) Y
\end{aligned}
$$

with $Y=\frac{1}{2} M \xi$;

$$
\begin{aligned}
u u_{20,2}^{\mathrm{I}}(\xi)= & -\frac{a}{3(2 N \tan (N \xi)+p)}\left[8 N^{3} \tan (N \xi) \xi\right. \\
& \left.+4 p N^{2} \xi+12 q r\right]+d_{1}
\end{aligned}
$$

where $\xi=a x+y+c z-\left(\frac{1}{2} a^{3} p^{2}-2 a^{3} q r-\frac{3}{2} c\right) t, d_{1}$ is an arbitrary constant.

Remark 3.2 All of the solutions presented in Section 3 have been checked with Maple 17 by putting them back into the original equations and the number of arbitrary constants has been reduced to a minimum.

Remark 3.3 Most of the solutions presented in Section 3 (e.g., (3.16), (3.17), (3.20) and (3.27)) cannot be obtained by means of the Riccati equation expansion method [23] and its existing improvements like the one in [24]. To the best of our knowledge, they have not been reported in literature.

\section{Conclusions}

In this paper, we used and further developed Zhang and Peng's modification of the Fan sub-equation method, and many exact solutions of the $(2+1)$-dimensional GCBS equation and the $(3+1)$-dimensional GBLMP equation were successfully found out. The obtained results show the effectiveness and advantages of the modified Fan sub-equation method in handling the solution process of high-dimensional NLPDEs.

It is necessary to point out that in Step 3, to make the work feasible, how to choose appropriately variables in the ansatz would be the key step in the computation to find out a useful solution of the algebraic equations.

It is interesting to note that research on solving fractional PDEs has attracted much attention recently, and there have been some new developments especially in solving time-fractional PDEs of the Fan sub-equations method (e.g., [25, 26]). However, searching for exact analytical solutions of nonlinear fractional PDEs is still on a preliminary stage. It is difficult to extend the methods for NLPDEs to fractional PDEs. Very recently, the known variable separation method has been extended to such fractional PDEs with initial 
and boundary conditions [27]. So, extending the work in the present paper is worthy of study.

\author{
Acknowledgements \\ The first author wishes to thank Dongming Wang for his encouragement and profound concern. The authors also wish to \\ thank the reviewers for their valuable comments that have helped to improve the presentation of the paper.
}

\title{
Funding
}

The project was supported by open fund of Guangxi Key laboratory of hybrid computation and IC design analysis (HCIC 201602).

\section{Competing interests}

The authors declare that they have no competing interests.

\section{Authors' contributions}

The authors contributed equally to this paper. All authors read and approved the final manuscript.

\section{Publisher's Note}

Springer Nature remains neutral with regard to jurisdictional claims in published maps and institutional affiliations.

Received: 23 June 2017 Accepted: 7 December 2017 Published online: 22 January 2018

\section{References}

1. Toda, K, Yu, S, Fukuyama, F: The Bogoyavlenskii-Schiff hierarchy and integrable equations in $(2+1)$ dimensions. Rep. Math. Phys. 44, 247-254 (1999)

2. Gilson, C, Nimmo, J-J: A (2 + 1)-dimensional generalization of the akns shallow-water wave-equation. Phys. Lett. A $180,337-345(1993)$

3. Luo, L: New exact solutions and Bäcklund transformation for Boiti-Leon-Manna-Pempinelli equation. Phys. Lett. A 375, 1059-1063 (2011)

4. Ablowitz, M, Clarkson, P: Solitons, Nonlinear Evolution Equations and Inverse Scattering. Cambridge University Press, New York (1991)

5. Miurs, M: Bäcklund Transformation. Springer, Berlin (1978)

6. Matveev, V: Darboux Transformation and Solitons. Springer, Berlin (1991)

7. Fan, E: Darboux transformation and soliton-like solutions for the Gerdjikov-Ivanov equation. J. Phys. A, Math. Gen. 33, 6925-6933 (2000)

8. Tam, H-W, Ma, W-X, Hu, X-B, Wang, D-L: The Hirota-Satsuma coupled KdV equation and a coupled Ito system revisited. J. Phys. Soc. Jpn. 69, 45-52 (2000)

9. Parkes, E, Duffy, B: An automated tanh-function method for finding solitary wave solutions to non-linear evolution equations. Comput. Phys. Commun. 98, 288-300 (1996)

10. Fan, E: Extended tanh-function method and its applications to nonlinear equations. Phys. Lett. A 277, $212-218$ (2000)

11. Lü, Z, Zhang, H: On a new modified extended tanh-function method. Commun. Theor. Phys. 39, 405-408 (2003)

12. Fan, E, Dai, H: A direct approach with computerized symbolic computation for finding a series of traveling waves to nonlinear equations. Comput. Phys. Commun. 153, 17-30 (2003)

13. Fan, E: Uniformly constructing a series of explicit exact solutions to nonlinear equations in mathematical physics. Chaos Solitons Fractals 16, 819-839 (2003)

14. Zayed, E, Gepreel, K: The ( $\left.G^{\prime} / G\right)$-expansion method for finding traveling wave solutions of nonlinear pdes in mathematical physics. J. Math. Phys. 50, 013502 (2009)

15. Zhang, Y: Solving STO and KD equations with modified Riemann-Liouville derivative using improved $\left(G^{\prime} / G\right)$-expansion function method. IAENG Int. J. Appl. Math. 45, 16-22 (2015)

16. Wang, $M, L i$, X: Extended $F$-expansion and periodic wave solutions for the generalized Zakharov equations. Phys. Lett. A 343, 48-54 (2005)

17. Yomba, E: The extended Fan's sub-equation method and its application to KdV-MKdV, BKK and variant Boussinesq equations. Phys. Lett. A 336, 463-476 (2005)

18. Soliman, A, Abdou, M: Exact travelling wave solutions of nonlinear partial differential equations. Chaos Solitons Fractals 32, 808-815 (2007)

19. Zhang, S, Peng, A: A modification of fan sub-equation method for nonlinear partial differential equations. Int. J. Appl. Math. 44, 10-14 (2014)

20. Xie, F, Zhang, Y, Lü, Z: Symbolic computation in non-linear evolution equation: application to $(3+1)$-dimensional Kadomtsev-Petviashvili equation. Chaos Solitons Fractals 24, 257-263 (2005)

21. Sirendaoreji, Jiong, S: Auxiliary equation method for solving nonlinear partial differential equations. Phys. Lett. A 309, 387-396 (2003)

22. Wu, W: Algorithms and Computation. Springer, Berlin (1994)

23. Zhu, W, Ma, S, Fang, J, Ma, Z, Zhu, H: Fusion, fission, and annihilation of complex waves for the $(2+1)$-dimensional generalized Calogero-Bogoyavlenskii-Schiff system. Chin. Phys. B 23, 060505 (2014)

24. Li, B, Chen, Y: Exact analytical solutions of the generalized Calogero-Bogoyavlenskii-Schiff equation using symbolic computation. Czechoslov. J. Phys. 54, 517-528 (2004)

25. Zhang, S, Zhang, H-Q: Fractional sub-equation method and its applications to nonlinear fractional pdes. Phys. Lett. A 375, 1069-1073 (2011) 
26. Zhang, S, Cai, B, Xu, B: Variable separation method for nonlinear time fractional biological population model. Int. J. Numer. Methods Heat Fluid Flow 25, 1531-1541 (2015)

27. Zhang, S, Hong, S: Variable separation method for a nonlinear time fractional partial differential equation with forcing term. J. Comput. Appl. Math. (2017). https://doi.org/10.1016/j.cam.2017.09.045

Submit your manuscript to a SpringerOpen ${ }^{\circ}$ journal and benefit from:

- Convenient online submission

$\checkmark$ Rigorous peer review

- Open access: articles freely available online

- High visibility within the field

Retaining the copyright to your article

Submit your next manuscript at $\gg$ springeropen.com 